\title{
Comparison of SNR Values for Different Wavelet Functions using Wavelet Packet Transformation
}

\author{
Dr Afroz ${ }^{1}$, Imtiyaz Ahmad Wani ${ }^{2}$ \\ Department of Mathematics ${ }^{1,2}$, MANUU, Hyderabad, India ${ }^{1,2}$ \\ Email: afroz.ahmad@manuu.edu.in ${ }^{1}$,wanimtiyaz85@gmail.com ${ }^{2}$
}

\begin{abstract}
In recent years an important contribution to the world of science has been electrocardiogram (ECG). It is no doubt an important Signal in biomedical science. This ECG is being analysed through a particular processing of wavelet transformation. It offers a reliable and valuable source of information for the expertise. It further adds the information regarding the heart disease. Among scores of techniques that are used for its analysis, wavelet transformation produces the best result and is very much acute in the diagnosis of cardiac arrhythmias. However, it needs an ECG with a minimum possible noise trapped during the processing of ECG. Recognising the importance of different methods that have been used so far, the paper intends to propose an algorithm for denoising the process of ECG. It is cogent to mention that the conduct of SNR values are noticed based on three mathematical formulae derived from an increasing function associated with threshold values. A comparison of SNR values between synthesis ECG and corresponding details coefficients is also discussed. Finally a comparison of SNR values obtained on using Haar Wavelet, Symlet-2, db-3, coef-2 and bior-1.3 wavelet in the proposed algorithm is shown. This algorithm is performed on a sample of ten ECG provide by MIT BIH Arrhythmias data base through an important transformation method namely Wavelet Packet Transformation.
\end{abstract}

Keywords-Wavelet transformation, Wavelet functions, thresholding, wavelet packet transformation

\section{INTRODUCTION}

An important and primary issue that occurs during recording of an ECG is noise addition in it[1].Therefore the morphological structure of an ECG gets disturbed and affects in proper diagnosis of diseases due to the following types of noises: powerline interference, baseline wander, electromyography noise, additive white Gaussian noise (AWGN) etc. Hence denoising ECG signal is of primary importance. In past, lot of techniques were applied in this aspect which banked upon type of the noise in ECG and the characteristics of noise. Wavelet domain filtering, empirical mode decomposition (EMD) based thresholding, artificial neural network[2], genetic algorithms[3], and other methods. One cardiac cycle of ECG signal consists of the P, Q, R, S and T waves along with some intervals and QRS Complex. QRS complex originally developed by Pan and Tompkins[4] The drawback in their algorithm was that the frequency variation in QRS complex negatively affects their performance. Therefore Hamilton and Tompkins developed a real time QRS detection algorithm in the $\mathrm{C}$ language[5]. Band pass filtering and temporal filtering [6] were also applied for QRS complex detection but did not provide simple way of selecting bandwidth of the filter and width of sliding window [7].
Wavelet analysis is an important tool which gives good estimation of signal in time frequency domain as well as its localization behaviour. Mostly wavelet based techniques are preferred for denoising a signal (like ECG) [8], [9], [10], [11]. The idea behind the wavelet transformation for denoising the signal is that it represents the signal into two types of coefficients[12]. The large magnitude coefficients represent the signal and the large amount of smaller magnitude coefficients represents noise. The wavelet coefficients produced due to wavelet decomposition process is a function of scale and position. Scale is of wavelet function which allows the process to capture fast activity and sharp spikes such a process is called scaling. Shift means passing it across the signal over time axis, known as translation. Therefore wavelet works in two ways viz., translation and scaling, due to less flexibility of discrete wavelet transformation in frequency plane and analysis of a signal for a specified frequency bandwidth. Coifman, Meyer and Wicherhauser [19] contributed their works. The subject for analysis in this paper is obtained from MIT-BIH Arrhythmia data base which is good and free source available for researchers in doing their respective research in their concerned field of specialization. In this data base on can select the record with varying time duration like 10 seconds or in minutes. Also each record has sampling frequency equal to $360 \mathrm{~Hz}$. 


\section{Available online at www.ijrat.org}

\section{PARAMETERS TO BE USED IN THIS PAPER}

\subsection{Wavelet}

Wavelet is a mathematical function or a small wave which cuts a signal into different frequency components. The general wavelet function is defined as

$$
\psi_{s, \tau}(t)=\frac{1}{\sqrt{|s|}} \psi\left(\frac{t-\tau}{s}\right)
$$

where $\psi \in L^{2}(R)$ is called mother wavelet and this set forms an orthonormal wavelet basis in the function space $L^{2}(R)$ ' $\tau$ ' is called shifted parameter that represents the location of the window on time axis i.e. it represents part of the signal which is being analysed and ' $\mathrm{s}$ ' is a scaling factor. The conditions of a function to be mother wavelet are as follows:

(1) A wavelet should be of finite energy i.e.

$$
E=\int|\psi(t)|^{2}<\infty
$$

(2) A mother wavelet is a function of zero average[14] i.e.

$$
\int_{-\infty}^{\infty} \psi(t) d t=0
$$

(3) The Fourier transform of $\psi$ at $\omega=0$ is Zero [15] i.e.

$$
\hat{\psi}(\omega=0)=\int_{-\infty}^{\infty} \omega(t) d t=0
$$

Wavelets are perfectly reconstruction function with linear and higher order polynomial shapes such as rectangle, triangle, $2^{\text {nd }}$ order polynomial etc. therefore wavelets are able to denoise the particular signal better than conventional filters. Different wavelets have different characteristics but there exist no universal rule in selecting a particular type of wavelet. ECG signal is non-stationary signal which demands wavelet for its analysis. It also has linear phase shift which also demands a wavelet to be symmetrical function. Since QRS has highest slope having characteristics in its shape and event is localized in time which also makes sense of applying wavelets in its analysis. In this paper we are going to use Haar wavelet, Symlet wavelet, db-3, Coef-2 which are orthogonal wavelet and one biorthogonal wavelet namely Bior-1.3 wavelet which are symmetrical and near symmetrical in nature. Also the experimentation is carried out for denoising of ECG signal in presence of power- line interference. Here the wavelets have no explicit expression except for Haar Wavelet. The Haar Wavelet is defined as

$$
\psi(x)=\left\{\begin{array}{cc}
1 ; & 0 \leq x<1 / 2 \\
-1 ; & 1 / 2 \leq x<1 \\
0 ; & \text { otherwise }
\end{array}\right.
$$

The scaling function of this Haar Wavelet is

$$
\phi(x)=\left\{\begin{array}{lc}
1 ; & 0 \leq x<1 \\
0 ; & \text { otherwise }
\end{array}\right.
$$

\section{TRANSFORMATION USED IN THIS PAPER}

\subsection{Continuous Wavelet Transformation}

The continuous wavelet transformation of a signal $f(t)$ is defined as [16]

$$
W_{f}(s, \tau)=\frac{1}{\sqrt{s}} \int_{-\infty}^{\infty} f(t) \stackrel{*}{\psi}\left(\frac{t-\tau}{s}\right) d t
$$

Here $\psi$ is the wavelet function. The complex conjugate $*$ is used only if wavelet function is complex function otherwise not. Also's' and ' $\tau$ ' are defined in 2.1. As mentioned in introduction section that wavelet transformation is one of the best approaches for denoising signal. Here in CWT a signal (continuous function) is divided into wavelets i.e. in terms of inner product CWT is actually inner product of a time varying signal $\mathrm{f}(\mathrm{t})$ and set of wavelets $\psi_{\mathrm{S}, \tau}(\mathrm{t})$ given in [17] as

$$
\mathrm{W}_{f}(\mathrm{~s}, \tau)=\left\langle\mathrm{f}, \psi_{\mathrm{s}, \tau}\right\rangle=\frac{1}{\sqrt{\mathrm{s}}} \int \mathrm{f}(\mathrm{t}) \psi^{*}\left(\frac{\mathrm{t}-\tau}{\mathrm{s}}\right) \mathrm{dt}
$$

where ' $s$ ' is a scale factor and ' $\tau$ ' is a translation factor. Also $*$ is a complex conjugate. $\psi$ is called mother wavelet and it generates a family of functions known as daughter wavelet by scaling and translation of $\psi$. The mother wavelet is given by

$$
\psi_{\mathrm{s}, \tau}(\mathrm{t})=\frac{1}{\sqrt{\mathrm{s}}} \psi\left(\frac{\mathrm{t}-\tau}{\mathrm{s}}\right)
$$

where $s>0$. If $s>1$ then wavelets are dilated and if $s<1$ then wavelets are contracted. The constant $\frac{1}{\sqrt{\mathrm{s}}}$ is called normalization constant. 


\section{Available online at www.ijrat.org}

\subsection{Discrete Wavelet Transformation}

Discrete wavelet transformation is a wavelet transformation where wavelets are discretely sampled. i.e. DWT is in fact the continuous wavelet transformation but with discrete scale and translation. So, the wavelet transformation is evaluated on discrete values of scale and translation defined as $\mathrm{s}=\mathrm{s}_{0}^{i}$ and translation $\tau=\mathrm{k} \tau_{0} \mathrm{~s}_{0}^{i}$, where $\mathrm{i}$ and $\mathrm{k}$ are integer and $\mathrm{s}_{0}>1$.The discrete wavelets are defined mathematically:

$$
\begin{aligned}
& \psi_{\mathrm{i}, \mathrm{k}}(\mathrm{t})=\left(s_{0}\right)^{\frac{-i}{2}} \psi\left(\mathrm{s}_{0}^{-i}\left(\mathrm{t}-\mathrm{k} \tau_{0} \mathrm{~s}_{0}^{i}\right)\right. \\
& \text { Or } \quad \psi_{\mathrm{i}, \mathrm{k}}(\mathrm{t})=\left(s_{0}\right)^{\frac{-i}{2}} \psi\left(\mathrm{s}_{0}^{-1} \mathrm{t}-\mathrm{k} \tau_{0}\right)
\end{aligned}
$$

Here we note that the discrete wavelet depends on $\mathrm{s}_{0}$ and $\tau_{0}$. When $\mathrm{s}_{0}$ is close to 1 and $\tau_{0}$ is small then discrete wavelets behave like continuous wavelets. The discrete wavelet transformation for a signal (function) $f(x)$ is given by:

$$
\mathrm{W}_{\mathrm{f}}(\mathrm{i}, \mathrm{k})=\int \mathrm{f}(\mathrm{t}) \cdot \psi^{*}{ }_{(\mathrm{i}, \mathrm{k})}(\mathrm{t}) \mathrm{dt}=\left\langle\mathrm{f}(\mathrm{t}), \psi_{\mathrm{i}, \mathrm{k}}\right\rangle
$$

Where $f$ can be reconstructed by mathematical formula from discrete wavelet transform as given below

$$
f(t)=A \sum_{i ~ k} \sum_{f}(i, k) h_{i, k}(t)
$$

Here $A$ is a constant independent of signal $f(t)$.

Since discrete wavelet transformation is discretization of continuous wavelet transformation. DWT decomposes the frequency plane into dyadic bandwidths with resolution in exponential form. But this transformation is not flexible in analysing signal for a particular frequency bandwidth. Wavelet packet transformation is generalization of wavelet transformation. This concept was introduced by Coifman, Meyer and Wickerhauser [13] in which both approximation and detail coefficients are further decomposed into approximation and details at each level that is not the case in wavelet transformation decomposition. Because it only decomposes the signal into approximation and details at the very first level then on subsequent level only approximation is decomposed further into new approximation and details. Doing this has chances of losing information of the signal incorporated into details. That could be more important in making the expected inferences of the given signal for the investigation. Below diagrams shows the difference between the wavelet decomposition and wavelet packet decomposition.

So, in this paper we make use of wavelet packet transformation on the subject of analysis provided by MIT-BIH available on physio.net. Wavelet packet transformation is the generalization of Discrete Wavelet Transformation. It is more flexible tiling of the frequency plane arises greatly in case of analyzing for specified frequency bandwidth. This concept was brought by Coifman, Meyer and Wickerhauser [18], [19].

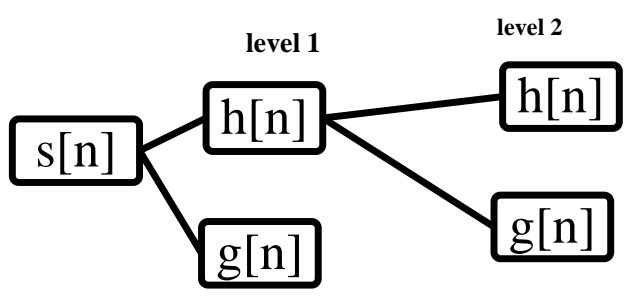

Fig. 1. Wavelet Decomposition $\mathrm{h}[\mathrm{n}]$ is Approximation and $\mathrm{g}[\mathrm{n}]$ is detail

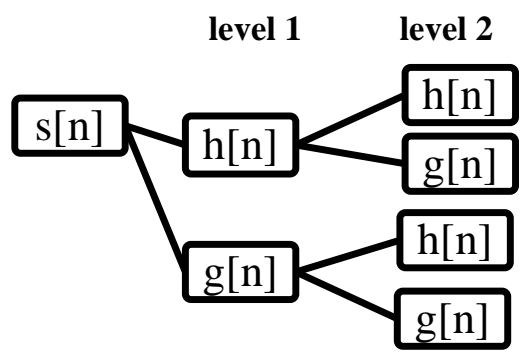

Fig. 2. Wavelet Decomposition $\mathrm{h}[\mathrm{n}]$ is Approximation and $\mathrm{g}[\mathrm{n}]$ is detail

\section{THRESHOLDING}

As we know signals carry noise with them. In order to remove noise from the signal we need some techniques which would help us to do so. In literature we do have some of the techniques[20] which helps us in denoising signal such as thresholding techniques 


\section{Available online at www.ijrat.org}

which includes hard thresholding[21], soft thresholding[21], hybrid thresholding[22], sure shrink thresholding[23] etc. In thresholding what we do is to remove small wavelet coefficients to zero which is actually noise present in the signal. This thresholding depends on a value $\mathrm{T}$ known as threshold value. If we take small value of $\mathrm{T}$ such that some coefficients which represents noise will still remain in the estimated signal. Then we don't get the proper denoised signal. In the similar fashion on taking large Value of $\mathrm{T}$ will remove some wavelet coefficients which do not belong to noise will also create a problem in getting denoised signal for proper analysis. Such a problem is shown by SNR values. More is the SNR value, better is the accuracy of denoising. So we need optimal threshold value of $\mathrm{T}$ which maximise the SNR. Let us define a function for threshold value as given below:

$$
\lambda(n)=\sigma \sqrt{n \log (N)}
$$

where $\mathrm{n}$ is natural number and $\mathrm{N}$ is sample size i.e. size of the signal. This function is an increasing function of ' $n$ '. if $n=2$, then

$$
\mathrm{T}=\lambda=\sigma \sqrt{2 \log (\mathrm{N})}
$$

Where $\mathrm{N}$ is length of the signal and $\sigma^{2}$ is the variance obtained in the finest level of detailed coefficients. Such a threshold value is known as Universal threshold value given in [24] For $n=1$ and 3 we get:

$$
\mathrm{T}=\sigma \sqrt{\log (N)}
$$

$\sigma^{2}$ is the variance obtained in the finest of detailed coefficients.

$$
\mathrm{T}=\sigma \sqrt{3 \log (\mathrm{N})}
$$

$\sigma$ is the standard deviation of the noise and can be calculated from median of detailed coefficients. In this paper we apply all of them separately and then take mean of the three and compare the results to each other.

\section{ALGORITHM}

Step 1. A noisy ECG signal is first decomposed with the help of wavelet transform at level 3 into approximation and detail coefficients.

Step 2. Obtained threshold value from detailed coefficients at finer level with the help formula mentioned above and then their mean for next step for the comparison purpose.

Step 3. Now apply Wavelet Packet Transformation for denoising the ECG signal at the threshold value obtained in step 2 .

Step 4. Reconstruct the denoised ECG signal by inverse wavelet packet transformation through MATLAB Wavelet Tool Box.

Step 5. Compare the results of SNR given by

$$
S N R=10 \log _{10} \frac{\sum_{i=1}^{N}(y(i)-x(i))^{2}}{\sum_{i=1}^{N}(\hat{x}(i)-x(i))^{2}}
$$

Where $y(i)$ and $x(i)$ are noisy ECG and Original ECG signal and $\hat{x}(i)$ is the estimated ECG signal.

\section{ANALYSIS}

Let us apply the algorithm for the sample ' $100 \mathrm{~m}$.mat' obtained from MIT-BIH ECG arrhythmia data base having 3600 samples/ signal and sampling rate $360 \mathrm{~Hz}$ with recording duration of 10 seconds.

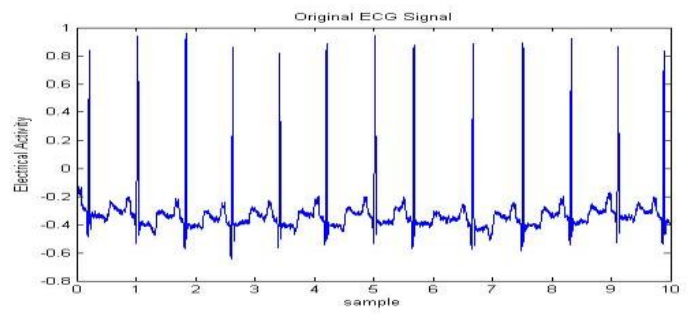

Fig. 3. Original Signal

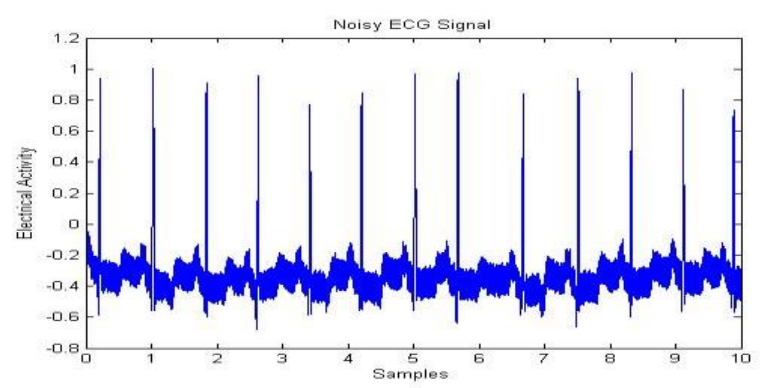

Fig. 4. Noisy ECG Signal 


\section{Available online at www.ijrat.org}

\section{ANALYSIS TABLES}

Table 1:

\begin{tabular}{|c|c|c|c|c|}
\hline \multicolumn{5}{|c|}{ Haar Wavelet } \\
\hline $\begin{array}{l}\text { Threshold } \\
\text { Values from } \\
\text { synthesised } \\
\text { ECG }\end{array}$ & $\sigma \sqrt{\log N}$ & $\sigma \sqrt{2 \log N}$ & $\sigma \sqrt{3 \log N}$ & $\begin{array}{c}\text { Average } \\
\text { threshol } \\
\mathrm{d}\end{array}$ \\
\hline SNR & 1.0874 & 0.7998 & 0.6778 & 0.8147 \\
\hline
\end{tabular}

Table 2:

\begin{tabular}{|c|c|c|c|c|}
\hline \multicolumn{5}{|c|}{ Haar Wavelet } \\
\hline $\begin{array}{l}\text { Threshold } \\
\text { Values from } \\
\text { Detail } \\
\text { Coefficient of } \\
\text { ECG }\end{array}$ & $\sigma \sqrt{\log N}$ & $\sigma \sqrt{2 \log N}$ & $\sigma \sqrt{3 \log N}$ & $\begin{array}{l}\text { Average } \\
\text { threshold }\end{array}$ \\
\hline \multicolumn{1}{|c|}{ SNR } & 0.9066 & 0.6825 & 0.5853 & 0.7248 \\
\hline
\end{tabular}

Table 3:

\begin{tabular}{|l|c|c|c|c|}
\hline \multicolumn{5}{|c|}{ Symlet-2 Wavelet } \\
\hline $\begin{array}{l}\text { Threshold } \\
\text { Values from } \\
\text { Detail } \\
\begin{array}{l}\text { Coefficient of } \\
\text { ECG }\end{array}\end{array}$ & $\sigma \sqrt{\log N}$ & $\sigma \sqrt{2 \log N}$ & $\sigma \sqrt{3 \log N}$ & $\begin{array}{c}\text { Average } \\
\text { threshold }\end{array}$ \\
\hline \multicolumn{1}{|c|}{ SNR } & 1.6143 & 0.4877 & 1.0857 & 0.8147 \\
\hline
\end{tabular}

Table 4:

\begin{tabular}{|l|c|c|c|c|}
\hline \multicolumn{5}{|c|}{ Symlet-2 Wavelet } \\
\hline $\begin{array}{l}\text { Threshold } \\
\text { Values from } \\
\begin{array}{l}\text { Detail } \\
\text { Coefficient of } \\
\text { ECG }\end{array}\end{array}$ & $\sigma \sqrt{\log N}$ & $\sigma \sqrt{2 \log N}$ & $\sigma \sqrt{3 \log N}$ & $\begin{array}{c}\text { Averag } \\
\text { e } \\
\text { thresho } \\
\text { ld }\end{array}$ \\
\hline \multicolumn{1}{|c|}{ SNR } & $\begin{array}{c}1.189 \\
1\end{array}$ & 0.8289 & 0.6995 & 0.8147 \\
\hline
\end{tabular}

It clearly shows from the analysis in the above four tables that more the threshold values lesser the SNR and all the tables indicate that as the value of $T \in\left(0, \frac{1}{2}\right]$, the value of $\mathrm{SNR}$ is good inside the interval than outside the interval from the right which is true as the noise in the signal is always lesser in the measurement than the signal values in the context of wavelet analysis. More importantly in above all tables denoising is shown better when standard deviation for getting threshold value is taken from synthesised signal than when standard deviation is taken from the details coefficients. Moreover thresholding is better through the threshold value taken from $\sigma \sqrt{\log N}$ than from $\sigma \sqrt{2 \log N}, \sigma \sqrt{3 \log N}$ and so on. On denoising the signal so obtained after this analysis looks like in the figure given below:

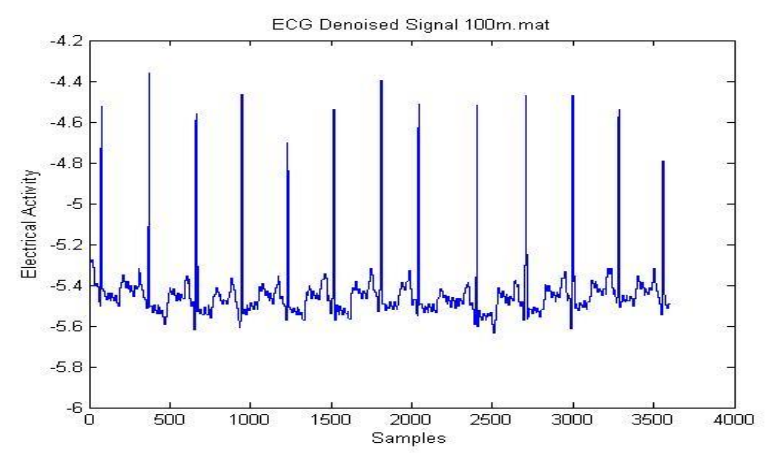

Fig. 5. Denoised ECG signal by above algorithm

On taking 10 samples from physio.net for analysis in which threshold value is taken from detail coefficients of each sample at level 3. The values of SNR shown in the table 5 is obtained as an average of three SNR values corresponding to three threshold values as obtained in table 2 and table 4 . 


\section{Available online at www.ijrat.org}

Table 5:

\begin{tabular}{|c|c|c|c|c|c|}
\hline Sample & $\begin{array}{c}\text { SNR by Haar } \\
\text { Wavelet }\end{array}$ & $\begin{array}{c}\text { SNR by } \\
\text { Symlet-2 } \\
\text { Wavelet }\end{array}$ & $\begin{array}{c}\text { SNR by db- } \\
\text { 3 Wavelet }\end{array}$ & $\begin{array}{c}\text { SNR by } \\
\text { Coef-2 } \\
\text { wavelet }\end{array}$ & $\begin{array}{c}\text { SNR by } \\
\text { Bior-1.3 } \\
\text { wavelet }\end{array}$ \\
\hline $100 \mathrm{~m} . \mathrm{mat}$ & 0.7249 & 0.9059 & 1.4440 & 1.0998 & 0.732 \\
\hline $101 \mathrm{~m} . \mathrm{mat}$ & 0.7587 & 1.2817 & 1.6290 & 1.6760 & 0.8719 \\
\hline $102 \mathrm{~m} . \mathrm{mat}$ & 1.1369 & 1.386 & 1.9163 & 2.3438 & 1.4205 \\
\hline $103 \mathrm{~m} . \mathrm{mat}$ & 0.3759 & 0.3725 & 0.8995 & 0.7236 & 0.2924 \\
\hline $104 \mathrm{~m} . \mathrm{mat}$ & 0.8553 & 1.3014 & 1.5527 & 1.6302 & 0.7141 \\
\hline $105 \mathrm{~m} . \mathrm{mat}$ & 0.5075 & 1.7076 & 3.5532 & 3.5547 & 0.6285 \\
\hline $106 \mathrm{~m} . \mathrm{mat}$ & 0.2559 & 0.4491 & 0.5063 & 0.8069 & 0.2955 \\
\hline $107 \mathrm{~m} . \mathrm{mat}$ & 0.1829 & 0.4754 & 0.9806 & 1.1563 & 0.1785 \\
\hline $108 \mathrm{~m} . \mathrm{mat}$ & 2.6592 & 5.4913 & 5.9593 & 5.2423 & 3.0738 \\
\hline $109 \mathrm{~m} . \mathrm{mat}$ & 0.683 & 2.2176 & 3.5282 & 3.8667 & 0.6954 \\
\hline
\end{tabular}

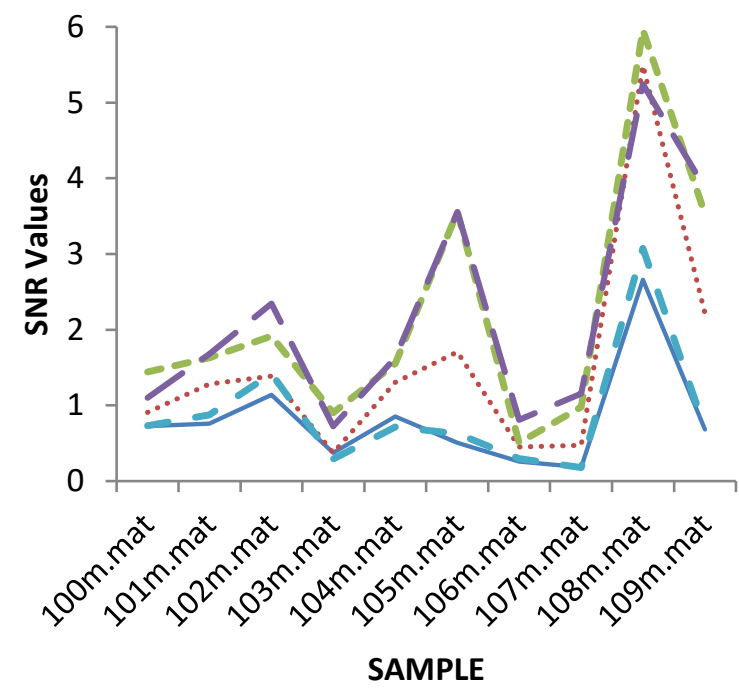

_ snr by Haar wavelet

........ snr by symlet wavelet

$-\infty$ snr by $\mathrm{db}-3$ wavelet

- snr by coef- 2

- snr by Bior-1.3 wavelet

Fig. 6. SNR Comparison in Scatter plot

The scatter plot of SNR values conveys that the algorithm works better in coef.-2 wavelet followed by db-2, symplet-2, bior-1.3 and Haar wavelet somehow in order. This means all the wavelets under discussion works in denoising the signal but varies in results. This is because of the property difference between them. Since coef.-2, symplet and db-3 shows better SNR results than Biorthogonal may be because later is biorthogonal in nature and rest including Haar wavelet are orthogonal in nature. Haar Wavelet shows lesser denoising than Bior-1.3 may be being discontinuous in nature. Finally one should keep properties of wavelet in view in the selection of wavelet for denoising of a signal and the properties of the signal itself.

\section{CONCLUSION}

In this paper, denoising algorithm is proposed and is tested on ten ECG samples provided by MIT BIH Arrhythmias database. Furthermore, a comparison of denoising is done by taking threshold values provided by mathematical functions used in different research works. Hence the results of some of the wavelet functions are compared at level 3. This comparison is shown in terms of SNR as an average of three SNR values obtained separately from given mathematical formulae by soft thresholding method. Finally denoising is found better in taking Coef-2 wavelet as a wavelet function in the algorithm than by taking other wavelets in this paper. The simulation of this whole paper is done in MATLAB. 


\section{Available online at www.ijrat.org}

\section{REFERENCES}

[1] G. D. Clifford, F. Azuaje, and P. E. McSharry. "Advanced Methods and Tools for ECG Analysis". 2006.

[2] A. Efros and T.Leug. "Texture Synthesis by Non Parametric Sampling". in In Proc. Ins. Conf. Computer Vision, volume 2, pp. 1033-1038, 1999.

[3] Y. Meyer. "Oscillating Patterns in Image Processing and Nonlinear Evoluation Equation".AMS Univerity Lect. Ser., vol. 22, 2002.

[4] J. Pan and W.J. Tompkins. "A real-time QRS Detection Algorithm”.IEEE Trans. Biomed. Eng., vol. 32, pp. 230-236, 1995.

[5] P. S. Hamilton and W.J.Tompkins. "Quantitative Investigation of QRS Detection Rules Using the MIT/BIH Aarythmia Database," IEEE Trans. Biomed. Eng., vol. 33, pp. 1157-1165, 1986.

[6] H. A. N. Dinh, D.K.Kumar, N.D.Pah, and P.Burton. "Wavelet for QRS Detection". in proceeding of the 23rd annual EMBS iternational conference, Turkey, 2001.

[7] N.V.Thakor, J. G. Webster and W.J.Tompkins, "Estimation of QRS Complex Power Spectra for Design of a QRS Filter," IEEE Trans. Biomed. Eng., vol. 31, pp. 702-706, 1986.

[8] A. P., "Wavelet Transform and the ECG: A review". Physiol Meas, vol. 26, pp. 155-199, 2005.

[9] B. Singh and A. Tiwari. "Optimal Selection of Wavelet Basis Function Applied to ECG Signal Denoising". Digital signal process, vol. 16, pp. 275-287, 2006.

[10] L.N.Sharma, S.Dandapat and A. Mahanta. "ECG Signl Denoising Using Higher Order Statistics in Wavelet Subbands".Biomed. Signal Process. Control, vol. 5, pp. 214-222, 2010.

[11] U. Seljuq, F.Himayun and H.Rashid. "Selection of an Optimal Mother Wavelet Basis Function for ECG Signal Denoising".IEEE Trans. Biomed. Eng., pp. 26-30, 2014.

[12] T. Sharma and K. K. Sharma. "QRS Complex Detection in ECG Signals Using Locally Adaptive Weighted Total Variation Denoising".Comput. Biol. Med., vol. 87, no. February, pp. 187-199, 2017.
[13] R.R.Coifman,Y.Meyer and V.Wickerhauser, "Wavelet analysis and signal processing".Wavelet Their Appl., pp. 453-470, 1992.

[14] R. S. Meena and A. Sharma. "Comparison and Analysis of Orthogonal and Biorthogonal Wavelets for ECG Comprssion”. no. 2, pp. 242 247, 2014.

[15] A. Mertins. "Signal Analysis: Wavelets, Filter Banks, Time-frequency transforms and applications”. 1999.

[16] S. PaulAddison. " The illustrated wavelet transform handbook”. Napier University, Edinburgh, UK, 2002.

[17] Y.sheng."Wavelet Transform Handbook”. CRC Press Inc, 1996.

[18] R. R. Coifman, Y. Mayer and V.Wickerhauser. "Wavelet Analysis and Signal Processing".Wavelet Their Appl., vol. 38, pp. 453-470, 1992.

[19]R. R. Coifman, Y. Meyer and M. V. Wickerhauser. "Wavelet Analysis and Signal Processing". in Wavelets and Their Applications, 1992, pp. 153-178.

[20] K. D. Priya, G. S. Rao and P. S. V. S. Rao. "Comparative Analysis of Wavelet Thresholding Techniques with Wavelet-wiener Filter on ECG Signal”.Procedia Comput. Sci., vol. 87, pp. 178 183, 2016.

[21]D. L. Donoho, "Denoising by Soft Thresholding".IEEE Trans. Biomed. Eng., vol. 41, no. MAY, pp. 613-627, 1995.

[22] Chmelka and J.Kozumplik. "Wavelet-based Wiener Filter for Electrocardiogram Signal Denoising".Comput. cardiol, vol. 32, pp. 771776, 2005.

[23] D.L.Donoho and I. M. Johnstone. "Adapting to Unknown Smoothness via Wavelet Shrinkage".J.Am.Statis Ass. L., vol. 90, pp. 1200 1224, 1995.

[24] R.T.Ogden "Essential Wavelets for Statistical Applications and Data Analysis".Boston, MA: Birkhäuser Boston, 1997 